\title{
Research and Design of Night Electronic Pet Clothing
}

\author{
Ningjie Zhao, Xuefei Li, and Yi Liu ${ }^{\text {a, * }}$ \\ Beijing Institute of Fashion Technology, No. 2 Sakura East Street, Chaoyang District, Beijing, China \\ amableliuyi@163.com \\ *corresponding author
}

Keywords: Arduino UNO, Smart clothing, Pet clothing, Infrared control, Vibration test

\begin{abstract}
With the rapid development of science and technology in China and the rapid growth of national economy, various smart fabrics and wearable devices have appeared in our life. Pet owners can also use open source hardware Arduino to design a wearable smart suit for their pets that is both beautiful and practical. The electronic outerwear introduced in this paper solves the problem of pet loss that occurs when the owner takes pet for a walk at night, so that the pet can also have a sense of existence in the dark environment, so that the same kind and human can find out in time. This subject mainly designed a night-walking cloth for pets. The main control board used is the UNO board of Arduino, which is composed of IR rit infrared remote control sensor, analog ambient light sensor, digital vibration sensor module, DFPlayer Mini player module, 3V LED lamp band and speaker horn. With the infrared remote controller as the general control, it can play music, display the pattern lighting, make visual output to the light intensity of the surrounding environment and light up with gait.
\end{abstract}

\section{Introduction}

With the opening of the 19th CPC national congress, the state leaders pointed out that major social contradictions have changed, and consumers' demand for clothing is not only a simple function of covering the body and keeping warm, but also a higher standard and demand for clothing's comfort and intelligence. In the era of constant development of electronic information technology, the combination of technology and traditional textile technology, the birth of smart clothing can meet these demands. Smart clothing can not only meet the ordinary wear effect, but also achieve more functions according to the designer's design concept.

With the development of economy and the improvement of people's living standards, pets have become people's emotional sustenance. Many people choose to keep pets to make up for their poor interpersonal relationship and emotional and spiritual deficiency caused by excessive pursuit of material benefits. Although animals are protected by their fur and fat, they also need clothing to help them maintain a comfortable temperature in especially cold weather. Although animals are protected by their fur and fat, they also need clothing to help them maintain a comfortable temperature in especially cold weather. This is not only to add warmth to pets, but also to respect pets and show their care. In addition, designing clothes for pets can not only make the owner happy, but also add new fun to the pet's life.

\section{General Design of the System}

\subsection{System Function Introduction}

This system mainly realizes the software design and hardware design of night pet coat, and the design content mainly includes: infrared remote control program, various visual output effect program of light, mp3 music play program, gait detection program, induction environment light intensity and weakness program, etc. Take Arduino as the control core to realize the basic visual output function of the night pet electronic coat. The hardware design includes infrared remote control, vibration sensor, music player and so on. 
The design of this system adopts functional modularization design method, which is mainly divided into three parts: integral design, functional module design and costume design.

\subsection{System Application Environment}

Arduino is a hardware processing system, characterized by open source, which is based on microcontroller board and integrated development environment for programming. Arduino is simple, and beginners are easy to learn. Unlike microcontrollers running on other Windows operating systems, Arduino can run on any platform that includes Windows, Linux, and Macintosh. Arduino can be used to develop interactive interfaces that capture input information from different switch keys and sensors while controlling the output of various physical devices, including light strips and other devices. There are many microcontrollers and microcontroller platforms that are suitable for use in interactive system design. For example: Parallax Basic Stamp, Netmedia's BX-24, Phidgets, MIT's Handyboard and others provide similar functionality. All of these tools, you don't need to care about the cumbersome details of microcontroller programming, you are provided with an easy-to-use toolkit. Arduino also simplifies the process of working with microcontrollers, but Arduino is superior in many places compared to other systems.

\subsection{Overall Design}

\subsubsection{Project Content}

The design of this project is based on the Arduino platform. The project mainly includes four parts: Arduino UNO development board, Arduino IDE, infrared remote control, lamp belt and music play. As shown in figure 1.

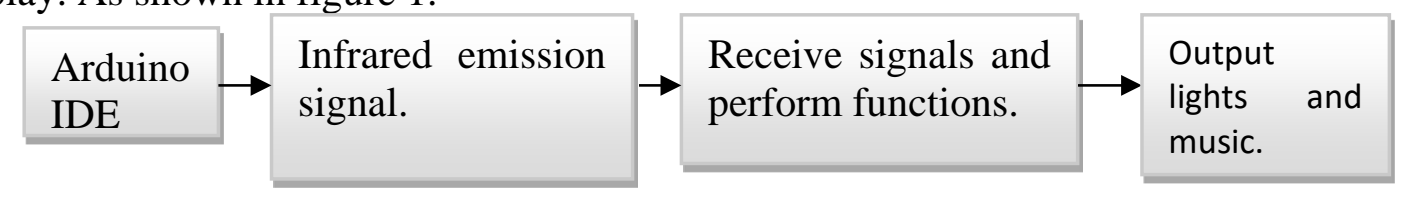

Figure 1 Project composition block diagram

\subsubsection{Function Design}

Arduino's features include: open source, cheap, easy to operate, and cross-platform, which has led to the rapid development of it, the first choice of people to learn about microcontrollers. This project selects Arduino as the platform, connects all the sensors together through the Arduino UNO board, and then realizes infrared control, gait detection, light induction, music play and visual output of lighting through software programming.

Overall function introduction: this system mainly realizes the software design and hardware design of night pet coat, and the design content mainly includes: infrared remote control program, various visual output effect program of lighting, mp3 music play program, gait detection program, induction environment light intensity and weakness program, etc. Take Arduino as the control core to realize the basic visual output function of the night pet electronic coat. The hardware design includes infrared remote control, vibration sensor, music player and so on. Finally, the circuit of the whole device is cut down, and the sewing is made on the inside of the designed clothes. The positions of each sensor and the output devices such as lamp belt and horn are fixed, and a complete device system is perfected. General function design block diagram, as shown in figure 2:

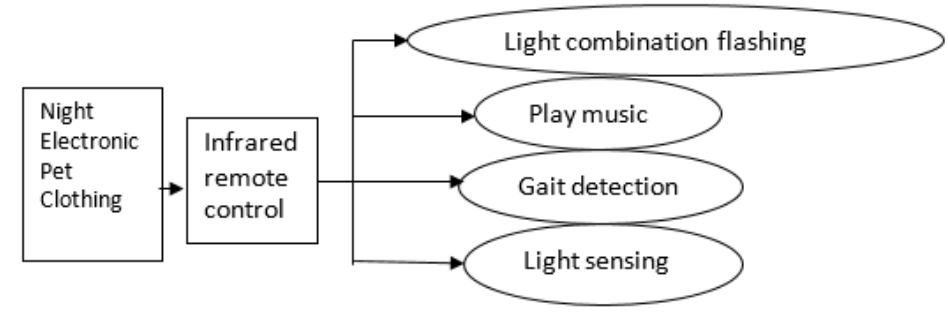

Figure 2 General design of the system 


\section{Circuit Diagram and Flow Chart}

\subsection{Analog Circuit}

TX and RX mp3 player module interfaces in TX and RX and UNO board connected to infrared sensors connected to the UNO board 11 digital pin position, photosensitive sensors connected to the analog pin A0 UNO board position, vibration sensors connected in UNO board of digital pins 2 position, can raise the interrupt 1 , six lights, respectively as the output termination in UNO board digital pins $4,5,6,7,8,9$. This is the circuit connection process of the whole device. In the actual connection process, a small size bread plate is used as expansion plate. It will also be connected to a small horn as the output device of the music module. The whole system is powered by a $9 \mathrm{~V}$ battery.

The entire unit is powered by an external 9V battery, sewing the entire device inside the garment. Sewing the shock sensor on the shoulder, because the joint part will move more frequently, which will make the sensitivity of the sensor better. The infrared sensor and the light sensor need to be exposed to the outside, so the two devices are sewn to the back of the garment to facilitate sensing of external sources of signal. The six outputs are replaced by light strips. The six strips of light surround the BIFT four letters and are evenly distributed throughout the sweater, highlighting the school's abbreviation, creating a colourful lighting effect. The small speaker connected to the mp3 module is sewn to the hem of the clothes, which can neither hinder the music playing nor affect the overall beauty.

\subsection{Software Design Process}

The software flow chart of the whole system design is shown in figure 3.

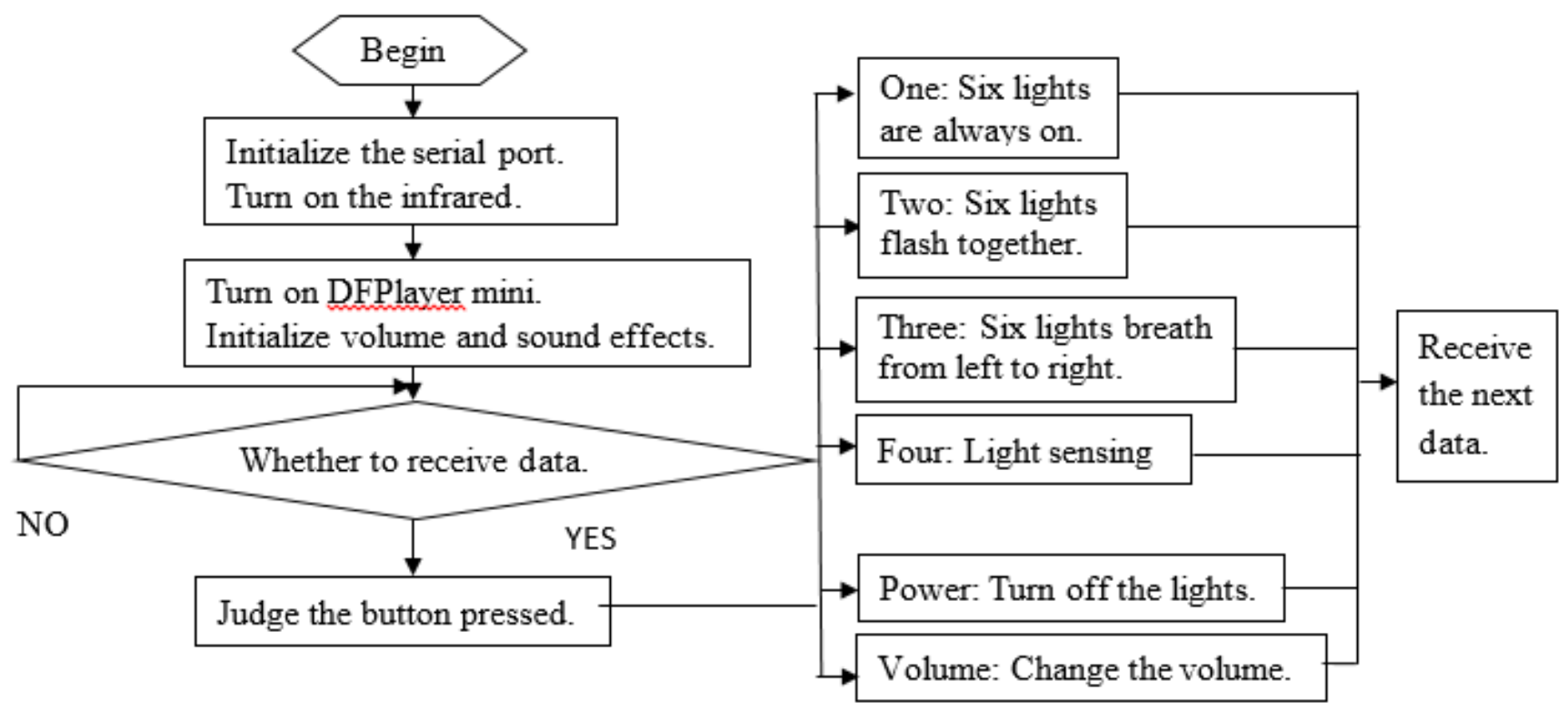

Figure 3 Software design process

\section{Modules Design}

Because the command code for each key is different, each command must be stored and executed according to the command code. Therefore, the program design adds a variable longPressKey to store the command code. The flowchart of the program is shown in figure 4 .

The program block diagram of infrared control is shown in figure 5. 


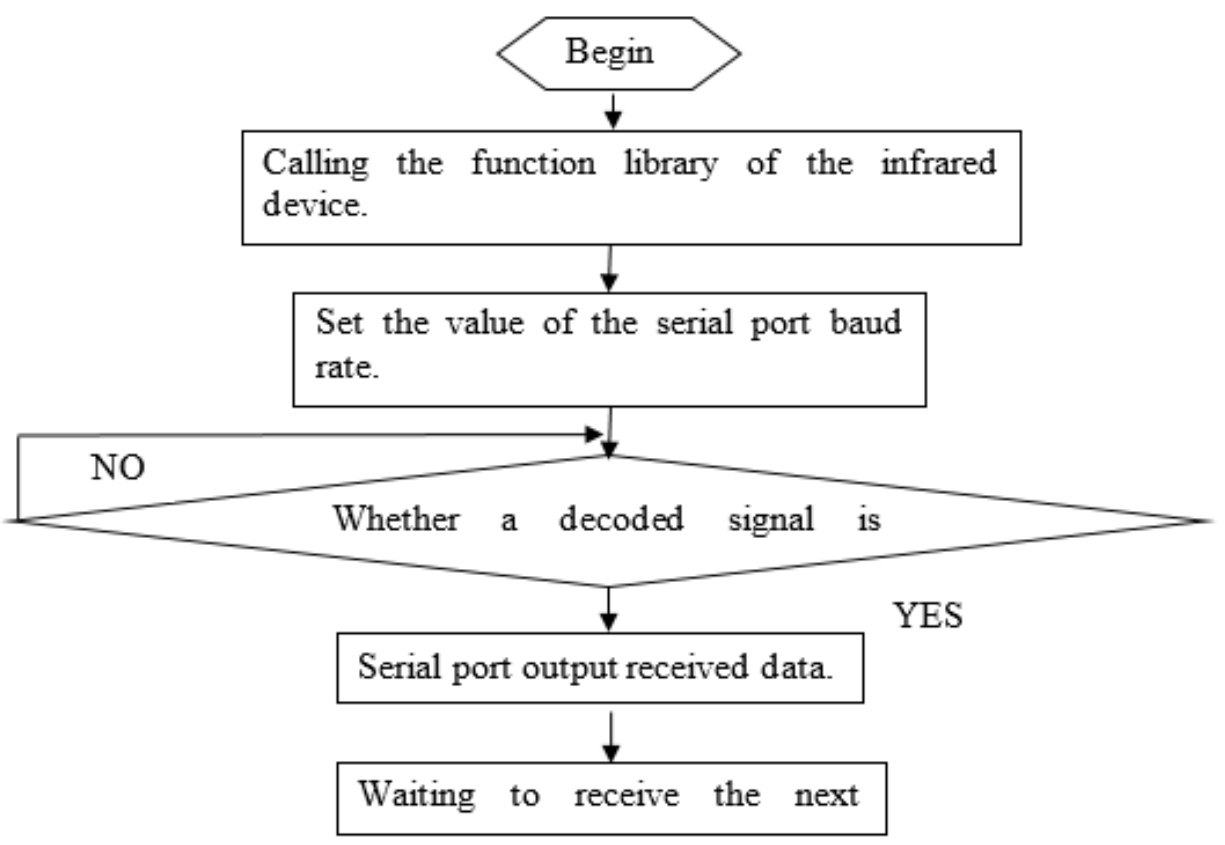

Figure 4 Infrared key value program flow

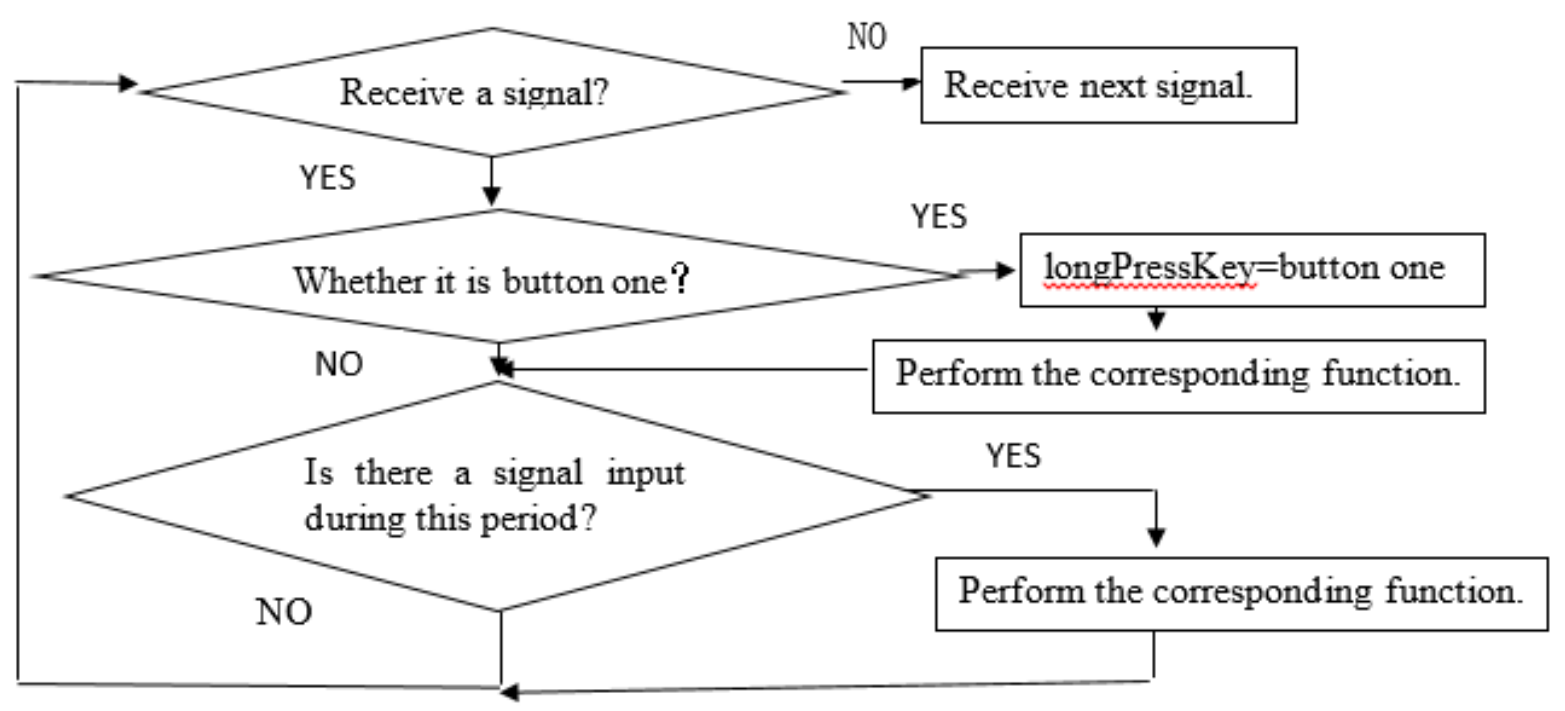

Figure 5 Infrared remote control flow chart.

\subsection{Vibration Sensor}

Connect the vibration sensor to the digital pin 2, break 0 . When the sensor is shaken, the lamp belt will light up. In the procedure used to interrupt function attachInterrupt (), when an external interrupt occurs, the interrupt function can play a role. Unlike other functions, which can be used as long as they are called, the pins it applies to can only be numeric pin 2 and 3, which represent interrupt pin 0 and 1 respectively. Here is a detailed introduction of interrupt function, which mainly includes three contents, interrupt number, function and mode:

Interrupt number: the interrupt number includes 0 and 1 , corresponding to the numeric pin 2 and 3, respectively.

Function: the interrupt function name of the call. When programming, take note:

1) When writing an interrupt function, the function cannot contain parameters and return values.

2) Since the function values of delay function and time function do not continue to change, these two functions cannot appear in the interrupt function.

3) Do not read the serial port in the interrupt function, because the data received by the serial port may be lost. 
Mode: interrupt condition. It only represents four specific situations: the first one, when the pin signal is low current, the second one, when the pin signal is high current, the third one, when the pin signal changes from low level to high level, the fourth one, when the pin signal changes from high level to low level.

\subsection{Light Sensor}

The name of the light sensor indicates that the device depends on the light. In dark environments, photoresistors have high resistance values. The stronger the light is, the bigger the resistance is, and conversely, it will be smaller. By reading this electrical resistance, we can detect different levels of light. The photosensitive resistance is connected to the analog port, and the analog value of the photosensitive diode is read, so the input and output mode need not be set. When the light is shone, the value of the simulation is reduced.

As the resistance value of both ends of the diode decreases, the voltage will also decrease.

The value of analog port $0 \sim 1023$ corresponds to the voltage value of $0 \sim 5 \mathrm{~V}$. The map () function is used here:

Map (value, from low, from high, to low, to high)

A map function is a function that maps a number from one scope to another. During programming, the minimum and maximum values of raw data and mapped data are required so that the raw data can be automatically mapped to the actual physical data using equation (1) when generating code:

$$
\mathrm{V}_{\text {map }}=\left(\mathrm{V}_{\text {raw }}-\mathrm{R}_{\min }\right) *\left(\mathrm{M}_{\max }-\mathrm{M}_{\min } \mathrm{R}_{\max }-\mathrm{R}_{\text {min }}\right)+\mathrm{M}_{\text {min }}
$$

Where Vmap is the data of the map, Vraw is the raw data, $\mathrm{R}_{\min }$ and $\mathrm{R}_{\max }$ are the minimum and maximum of the raw data. The minimum and maximum values of $\mathbf{M}_{\min }$ and $\mathrm{M}_{\max }$ mapping data.

In the program, ledlevel=map(sensorValue,0,1023,0,6), the analog value read from the light sensor is mapped to the range of six light bands, the value is assigned to the variable ledlevel, and then several light bands can be lit. Finally, the current light intensity is output visually by way of light brightness.

\subsection{Light Modes}

Eight lighting combination flashing modes have been designed in the system. The specific modes are shown in table 1.

Table 1 Light modes

\begin{tabular}{|l|l|}
\hline No. & The modes \\
\hline 1 & Six lights are always on. \\
\hline 2 & Six lights flash together. \\
\hline 3 & Six lights breathe separately. \\
\hline 4 & Six lights are sequentially changed in the order of the specified colors. \\
\hline 5 & $\begin{array}{l}\text { Six lights are quickly lit in order from left to right, and then turned off in order from } \\
\text { right to left, with a delay time of } 30 \text { ms between each light. }\end{array}$ \\
\hline 6 & $\begin{array}{l}\text { The three lights are grouped together, and the two groups are alternately lit with a } \\
\text { delay time of 380ms. }\end{array}$ \\
\hline 7 & $\begin{array}{l}\text { The two lights are grouped together, and the three groups are alternately lit with a } \\
\text { delay time of 100ms. }\end{array}$ \\
\hline 8 & Divide six lights into two groups and breathe in order. \\
\hline
\end{tabular}

\subsubsection{Breathing Light}

In the digital pins on Arduino UNO, there are six pins marked with wavy lines like " ", which means that the port has PWM function. PWM, which is a technology that can get analogy quantities. A square wave is formed through digital control. The square wave signal only switches two states, on and off. By controlling the ratio of opening and closing durations, you can simulate a voltage change from 0 to $5 \mathrm{~V}$. The time taken by the high level is called pulse width, so PWM is also called 
pulse width modulation. PWM was observed by the following five square waves, as shown in figure $6^{[5]}$.

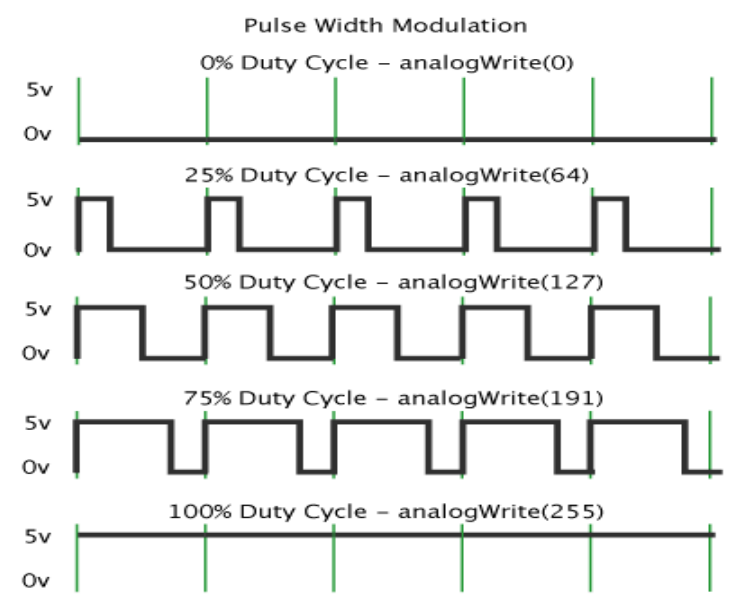

Figure 6 PWM Waveform

The green vertical line above represents a period of the square wave. Each value written in the analog Write(value) corresponds to a percentage, which is also called Duty Cycle. It refers to the percentage that the duration of high level over low level within a cycle is obtained. In the figure, from top to bottom, the first square wave has a duty ratio of $0 \%$ and the corresponding value is 0 . The LED has the lowest brightness, which is the state of extinction. The longer the high level lasts, the brighter it will be. So, the last one with a $100 \%$ duty ratio is 255 , which is the brightest LED. 50\% is the brightest half, and $25 \%$ is relatively dark. The value range is $0-255$, the voltage per unit is $5 / 255 \mathrm{v}$, the return value of analogRead is $0-1024$, and the voltage per unit is $5 / 1024 \mathrm{v}$. Digital pins with PWM function can use the analogy write (PIN, value) function to send the analogy values to the digital pins, so that the light can be controlled to have a slow process of gradually turning on and off, which feels like breathing evenly.

\subsubsection{Thunder Light}

Using the digitaloutput function of digitaloutput, in the for loop(), light up sequentially from left to right, and then dark from right to left, execute continuously and circularly. Here is a detailed description of several of these styles of subroutines:

Style 1 subroutine: the led first starts from the leftmost light, then lights up six led lights after a distance of $30 \mathrm{~ms}$, and then lights out six led lights after a distance of $30 \mathrm{~ms}$ from the rightmost light.

Style 2 subroutine: change the parameter value, and divide the six lamps into two groups. Turn on, set delay of $100 \mathrm{~ms}$ in the middle, and then turn off. The delay time is also $100 \mathrm{~ms}$.

Style 3 subroutine: change the parameter value again, divide the six lights into three groups, turn on, set delay of 500ms in the middle, and then turn off, the delay time is also 500ms.

The rest of the combined pattern lighting changes the number of lights on by a for cycle(), as well as the delay time, delay function, which delays the lighting time of each light, to change the effect of light flicker.

\subsection{Music Play}

DFPlayer has specialized library files < SoftwareSerial.h> and <DFPlayer_Mini_Mp3.h>.The connection of DFPlayer USES the TX and RX pins of the MP3 module. Here is a brief introduction of the relationship between USB, TX and RX:

TX and RX are the pins for serial communication, and USB is the universal serial bus protocol. Most computers now only USB port, the individual will have a serial port, and the Arduino using AVR chip, so the Arduino in order to solve the problem of computer doesn't have a serial port, use a USB serial adapter put the USB data into a serial port, and then received the TX and RX of Arduino, they both transmission of data is the same, just using different protocols. Serial. Read reads the data of RX pin corresponding register. The command of Serial. Read runs on the main chip. Similarly, 
Serial. Print is written on the corresponding register of TX pins.

\section{Overall Result}

Finally, all the circuit modules are combined and loaded into a simple fabric. Worn by a real dog running free on the night scene in the actual test. The entire device is controlled by the infrared remote control and is equipped with $9 \mathrm{~V}$ power supply. The specific functions are as follows:

Button 0: gait detection. 6 output lamp bands are on and off according to the vibration.

Button 1: 6 output lights on.

Button 2: 6 output light bands flashing together.

Button 3: 6 output lamp bands are turned on by breathing respectively.

Button 4: the light sensor. With the change of ambient light intensity, the 6 output light bands will gradually light up. The darker the environment is, the more light bands will be lit.

Button 5: 6 output lamp strips are changed in order of specified color.

Button 6: 6 output lamp strips are quickly lit in order from left to right and then put out in order from right to left. The delay time between each lamp strip is $30 \mathrm{~ms}$.

Button 73 output lamp strips are one set, and the two sets of lamp strips are turned on alternately with a delay time of $380 \mathrm{~ms}$.

Button 8 two output lamp strips are one group, three groups of lamp strips are turned on alternately, and the delay time is $100 \mathrm{~ms}$.

Button 9: divide 6 lamp strips into two groups and light up in turn according to the style of breathing lamp.

The FUNC button represents the function of music playback. First press the FUNC key and then press the number 1-9 to play the songs in the corresponding SD card. VOL+ and VOL- represent the increase and decrease of volume, PAUSE means PAUSE/play and EQ means select sound effect.

The power button controls six output lamp bands, which can be turned off at any time. Several modes can be switched at will.

In the process of physical display, I respectively took two ways of physical display and model display to show the whole installation. The physical demonstration process is the effect after the pet is actually dressed. The vibration-sensing effect is reflected in the light band on the pet when it starts running and jumping. When the pet is stationary, the light band is not on. When music is played, pets will run and jump to the rhythm when they hear the cheerful music. When they hear the soothing music, they will become quiet. In places with poor lighting, turn on the light sensor, and the number of light bands will light up with the dimness of the environment. The use of wool as the raw material for pet clothing can reduce the pet's discomfort during the process of dressing and undressing, as well as the degree of friction between clothes and fur in actual activities.

\section{Conclusion}

This project is mainly designed for pets to design a set of clothes for walking at night, integrating wireless control, music playback, gait detection, light sensing and other functions to achieve a fusion of technology and intelligence. In addition, by analysing the effects of different fabrics on pet skin, I designed a comfortable and beautiful intelligent clothing. Smart clothing is a multidisciplinary, multi-technology cross-integrated research direction. At present, China's research on smart clothing, including the formed technology, is still in the preliminary research stage, and there are relatively few related topics for its research and development.

Although this paper completed the pre-design idea, in the process of designing step by step and subsequent use, it is found that there are still many areas for improvement. For example, if the pet is still at rest during the night, it is impossible to identify the location of the pet, and whether a GPS positioning function should be added to the device. This problem requires further research and analysis.

This design is specially designed for pets to customize a set of night pet electronic outerwear, 
and smart clothing is more applied to humans. As technology continues to evolve, in just a few decades, there will be a variety of devices, clothing, and apparel that will interact with the wearer's nervous system to add to the definition of clothing. New content. In the future, there will be more and more novel costumes in our lives.

\section{Acknowledgement}

This research was financially supported by Beijing Institute of Fashion Technology College of Special Plan Young Top-notch Talent Project(BIFTBJ201803),Beijing Institute of Fashion Technology College Student Research Training Program (2018),Beijing Institute of Fashion Technology Teaching Reform and Innovation Key Program (ZDJG-1709),Fang Zhi Zhi Guang China National Textile And Apparel Council Higher Education Teaching Reform and Innovation Program (2017-08), and Open Project of Digital and Interactive Media Key Laboratory (KF2017-04).

\section{References}

[1] D'Ausilio A. Arduino: a low-cost multipurpose lab equipment.[J]. Behavior Research Methods, 2012, 44(2):305-313.

[2] Teikari P, Najjar R P, Malkki H, et al. An inexpensive Arduino-based LED stimulator system for vision research.[J]. Journal of Neuroscience Methods, 2012, 211(2):227-236.

[3] Romanuck J. Illuminated pet clothing: US, US20080163831 [P]. 2008.

[4] Kumar,Ayush. Home automation via Bluetooth using the Arduino Uno microcontroller [M], 2016

[5] DFRobot[EB/OL]. http://www.dfrobot.com.cn/

[6] Miura, Hiroaki C/o Nissan Co Ltdintellectual Property Dpt 1-1. CLOTH FOR ELECTRICAL DEVICE: EP, EP1978341 [P]. 2008.

[7] Soleimani M..Knitted switches for smart clothing using double electrode technology [J].Sensor Review, 2008, 28(3):229-232.

[8] Dina Meoli, Traci May-Plumlee. Interactive electronic textile development: A review of technologies [J].World Sport Activewear, 1999, 4(5):19-20.

[9] Miura H. Cloth for electric device: US, US 7886617 B2 [P]. 2011.

[10] Tanner M, Eckel R, Senevirathne I. Enhanced low current, voltage, and power dissipation measurements via Arduino Uno microcontroller with modified commercially available sensors.[C]// APS March Meeting. APS March Meeting Abstracts, 2016.

[11] Pauta J A A, Vélez E P, Serpa-Andrade L. Braille teaching electronic prototype[C]// IEEE International Autumn Meeting on Power, Electronics and Computing. IEEE, 2017:1-7.

[12] Teikari P, Najjar R P, Malkki H, et al. An inexpensive Arduino-based LED stimulator system for vision research.[J]. Journal of Neuroscience Methods, 2012, 211(2):227-236.

[13] Doukas C. Building Internet of Things with the Arduino [M]. 2012.

[14] Baraka K, Ghobril M, Malek S, et al. Low Cost Arduino/Android-Based Energy-Efficient Home Automation System with Smart Task Scheduling [M]. 2013. 